\title{
Tumores de glándula submandibular: Experiencia clínica del Hospital Guillermo Grant Benavente
}

\author{
Submandibular gland tumors: \\ Clinical experience of Guillermo Grant Benavente Hospital
}

\author{
Felipe Fredes $\mathbf{C}^{1}$, Mario Tapia $\mathbf{C}^{1}$, Joaquín Collao $\mathbf{C}^{2}$, Joaquín Ulloa $\mathbf{S}^{1}$, Álvaro Compan $\mathrm{J}^{1}$, \\ Alexis Urra $\mathbf{B}^{1}$, Pablo Ortega $\mathbf{R}^{1}$.
}

\begin{abstract}
RESUMEN
Introducción: Los tumores de la glándula submandibular son poco frecuentes. Representan menos del $1 \%$ de todos los tumores de cabeza y cuello. Corresponden al $10 \%$ de los tumores de las glándulas salivales. Aproximadamente el 50\% son malignos.

objetivos: Analizar la experiencia clínica con las submandibulectomías realizadas en el Servicio de Otorrinolaringología y Cirugía de Cabeza y Cuello del Hospital Guillermo Grant Benavente.

Material y método: Estudio descriptivo retrospectivo. Revisión de fichas clínicas de pacientes intervenidos de submandibulectomía entre el 01 de enero de 2011 y el 31 de diciembre de 2016 en nuestro servicio.

Resultados: Se realizaron 28 submandibulectomías por patología tumoral. La edad promedio fue de 54 años, siendo el $71 \%$ de sexo femenino. 21 tumores fueron benignos (75\%), siendo el más frecuente el adenoma pleomorfo (14 casos), y 7 tumores malignos (25\%), siendo el más frecuente el carcinoma adenoidequístico. Se reportaron dos complicaciones transitorias (7\%), una paresia de la rama marginal del nervio facial y una paresia del nervio hipogloso. El $42 \%$ de los tumores malignos presentó recurrencia.

Conclusión: Nuestra serie presenta resultados acordes a la literatura en cuanto a edad de presentación, distribución por sexo, tipos histológicos, tasa de complicaciones y recurrencia; sin embargo, difiere en la menor frecuencia de tumores malignos, siendo en nuestra serie de $25 \%$. Patología poco frecuente. La presencia de una masa indolora submandibular debe ser derivada a los centros de referencia para estudio y eventual resolución quirúrgica por el riesgo de malignidad.
\end{abstract}

Palabras clave: Glándulas salivales, tumores de la glándula submandibular, submandibulectomías.

\begin{abstract}
Introduction: Submandibular gland tumors are rare. They represent less than 1\% of all head and neck tumors. They correspond to $10 \%$ of the tumors of the salivary glands. Approximately $50 \%$ are malignant.
\end{abstract}

1 Médico del Servicio de Otorrinolaringología y Cirugía de Cabeza y Cuello, Hospital Guillermo Grant Benavente, Concepción, Chile.

2 Interno de Medicina, Universidad de Concepción, Chile.

Recibido el 16 de marzo, 2018. Aceptado el 23 de mayo, 2018. 
Aim: To analyze the clinical experience with the submandibulectomies performed in the Otorhinolaryngology and Head and Neck Surgery Service of the Guillermo Grant Benavente Hospital.

Material and Method: Retrospective descriptive study. Review of clinical records of patients treated with a submandibulectomy between January 1, 2011 and December 31, 2016 in our service.

Results: 28 submandibulectomies were performed due to tumor pathology. The average age was 54 years, being $71 \%$ female. 21 tumors were benign (75\%), being the most frequent the pleomorphic adenoma (14 cases), and 7 malignant tumors (25\%), being the most frequent the adenoidequistic carcinoma. Two transient complications (7\%) were reported, a paresis of the marginal branch of the facial nerve and a paresis of the hypoglossal nerve. $42 \%$ of malignant tumors presented recurrence.

Conclusion: Our series presents results according to the literature regarding age of presentation, distribution by sex, histological types, complication rate and recurrence; however, it differs in the lower frequency of malignant tumors, being in our series of $25 \%$. Uncommon pathology. The presence of a submandibular painless mass must be referred to the reference centers for study and possible surgical resolution due to the risk of malignancy.

Key words: Salivary glands, tumors of the submandibular gland, submandibulectomies.

\section{INTRODUCCIÓN}

Los tumores de glándulas salivales son poco frecuentes, representando entre el $1 \%$ al $3 \%$ de los tumores de cabeza y cuello ${ }^{1-3}$. Clásicamente el $80 \%$ se ubica en la glándula parótida, 10\%-15\% en la glándula submandibular, 5\%-10\% en glándulas salivares menores y menos del $1 \%$ en la glándula sublingual ${ }^{4}$. En el caso particular de las glándulas submaxilares, representan menos del $1 \%$ de los tumores de cabeza y cuello, y aproximadamente el $50 \%$ de ellos son malignos $(25 \% \text { a } 76 \%)^{5-12}$. Los tumores de la glándula submandibular habitualmente se presentan mediante un aumento de volumen indoloro en el triángulo submandibular. La presencia de dolor orienta a un proceso benigno como sialoadenitis y sialolitiasis. La parálisis del nervio lingual, hipogloso 0 marginal orienta a malignidad ${ }^{13}$. El estudio preoperatorio de los tumores submandibulares suele realizarse con exámenes imagenológicos (ecografía, tomografía computarizada y resonancia magnética) y con punción aspirativa con aguja fina (PAAF), sin embargo, el diagnóstico definitivo se realiza mediante biopsia de la pieza operatoria, siendo la submandibulectomía la técnica de elección ${ }^{14-17}$.

En este artículo se realiza un análisis de los tumores de la glándula submandibular operados entre el año 2011 y 2016 en el Servicio de Otorrinolaringología y Cirugía de Cabeza y Cuello (ORL-CyC), en el Hospital Clínico Regional de Concepción Guillermo Grant Benavente (HGGB).

\section{OBJETIVO}

Analizar la experiencia clínica con las submandibulectomías realizadas en el Servicio de ORL- CyC del Hospital Guillermo Grant Benavente (HGGB), y caracterizar a los pacientes afectados por patología tumoral de glándula submaxilar.

\section{MATERIAL Y MÉTODO}

Estudio retrospectivo, se realizó una revisión de todos los pacientes sometidos a submandibulectomía en el Servicio de ORL-CyC, del HGGB entre el 1 de enero del año 2011 y el 31 de diciembre de 2016. Se obtuvieron los datos a través de la Unidad de Estadísticas del Hospital y de los registros del pabellón quirúrgico. Se registraron los datos generales del paciente, presentación clínica, técnica quirúrgica, histología, complicaciones, recurrencia y resultados posoperatorios. En todas las cirugías 
el primer cirujano fue un médico staff del equipo de Cirugía de Cabeza y Cuello. Se excluyeron los pacientes operados por patología benigna no tumoral y aquellos operados por otros equipos de nuestro hospital, como cirugía maxilofacial y cirugía infantil.

\section{RESULTADOS}

En el periodo descrito, se realizaron 43 submandibulectomías. Se excluyeron 15 pacientes que fueron operados bajo el diagnóstico de sialoadenitis crónica (11 casos) y sialolitiasis (4 casos), todos confirmados con diagnóstico histológico. Finalmente fueron incluidos 28 pacientes operados por patología tumoral. El promedio de edad fue de 54,5 años (rango 29 - 83 años), 20 pacientes de sexo femenino $(71,4 \%)$ y 8 de sexo masculino $(28,6 \%)$. El motivo de consulta en el $100 \%$ de los casos fue el aumento de volumen submandibular indoloro. No se registraron parálisis de nervio lingual, hipogloso ni marginal. En 3 casos se evidenciaron adenopatías al examen físico. En todos los casos se realizó estudio preoperatorio con exámenes imagenológicos, 9 exclusivamente con ecografía, 15 con tomografía computarizada (TC) exclusivamente y en 4 casos se realizaron ambos exámenes. No se solicitó resonancia magnética. El principal hallazgo fue la presencia de un tumor único en la glándula submandibular. En 4 casos se evidenció la presencia de adenopatías. No se realizaron punciones aspirativas con aguja fina ni biopsias incisionales previo a la cirugía.

Todos los pacientes fueron sometidos a una submandibulectomía, en 4 casos $(14,2 \%)$ se asoció un vaciamiento cervical supraomohioideo ipsilateral en los casos con adenopatías sospechosas previa confirmación con biopsia rápida. En un caso se realizó el vaciamiento cervical en un segundo tiempo debido a que la biopsia rápida de la pieza operatoria informó en primera instancia ausencia de malignidad y el resultado diferido informó un carcinoma mucoepidermoide. El tiempo quirúrgico promedio fue de 98 minutos, 93 minutos cuando se realizó solamente la submandibulectomía, y 114 minutos cuando se asoció un vaciamiento cervical.

La estadía hospitalaria posoperatoria fue de 2,25 días (rango 1-6 días), todos fueron dejados con un drenaje aspirativo por 24 horas. No se reportaron hematomas cervicales ni infecciones de la herida operatoria, pero sí dos lesiones transitorias de nervio $(7 \%)$, un caso presentó una paresia de la rama marginal del nervio facial y otro presentó una paresia transitoria del hipogloso. En cuanto a la histología, 21 tumores fueron tumores benignos (75\%) y 7 malignos (25\%). Respecto a los tumores benignos (Figura 1), el más frecuente fue el

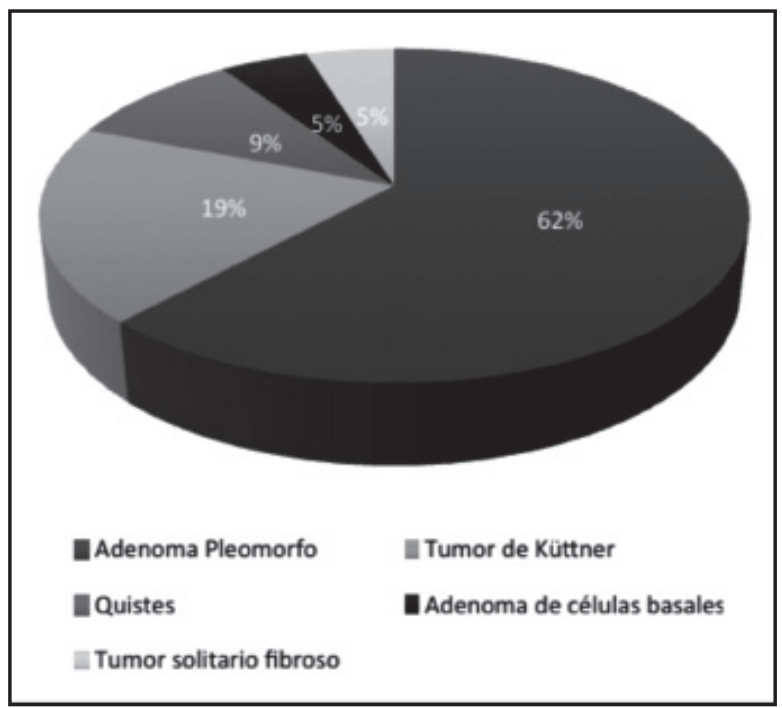

Figura 1. Histología tumores benignos de glándula submandibular. 
adenoma pleomorfo en 13 casos (62\%), seguido por cuatro tumores de Küttner (19\%), dos quistes infundibulares $(9 \%)$, un tumor solitario fibroso $(5 \%)$ y un adenoma de células basales (5\%).

En cuanto a los tumores malignos (Figura 2), el más frecuente fue el carcinoma adenoidequístico con tres casos $(43 \%)$, seguido por el carcinoma mucoepidermoide con dos casos $(29 \%)$, un carcinoma mioepitelial (14\%) y un melanoma (14\%). Respecto al tamaño tumoral, cuatro eran menores a $2 \mathrm{~cm}, 14$ tumores presentaban medidas entre 2 y $4 \mathrm{~cm}$, y 10 eran mayores a $4 \mathrm{~cm}$. Los menores a $2 \mathrm{~cm}$ fueron todos benignos, en el grupo de 2 a $4 \mathrm{~cm}$ se presentaron tres tumores malignos y en el grupo de tumores mayores a $4 \mathrm{~cm}$ cuatro eran malignos.

El seguimiento promedio fue de 19 meses, 15 meses en el caso de los tumores benignos y 39 meses en los malignos. La sobrevida en nuestra serie es de $96 \%$, con una sola defunción en un paciente con diagnóstico de tumor de Küttner que falleció producto de las complicaciones de un linfoma no Hodgkin a los 24 meses poscirugía. Los siete pacientes con tumores malignos fueron tratados con radioterapia complementaria. En el caso del carcinoma mucoepidermoide se realizó el vaciamiento cervical supraomohioideo ipsilateral en un segundo tiempo seguido de radioterapia y quimioterapia. Tres casos presentaron recurrencia, un carcinoma adenoidequístico presentó metástasis pulmonar a los 46 meses de seguimiento, estando actualmente en cuidados paliativos; el caso del carcinoma mioepitelial presentó una metástasis pulmonar a los 41 meses tratada con metastasectomía más radioterapia; y el caso del carcinoma mucoepidermoide presentó múltiples recidivas locales y en cavidad oral tratadas con cirugía y actualmente se encuentra en cuidados paliativos con metástasis pulmonares y hepáticas.

Al comparar el grupo de tumores benignos con el de tumores malignos, el primer grupo se caracterizó por ser pacientes de menor edad, con predominio de sexo femenino en relación 2:1, cirugías de menor duración, pero hospitalizaciones de similar duración que los malignos y la misma incidencia de complicaciones. Estas diferencias pueden verse en la Tabla 1.

\section{DISCUSIÓN}

Los tumores de la glándula submandibular son poco frecuentes, representan menos del $1 \%$ de los tumores de cabeza y cuello y alrededor del $10 \%$ de los tumores de glándulas salivales, esto varía según las distintas series entre $4 \%$ y $37 \%{ }^{5-12}$. En Chile,

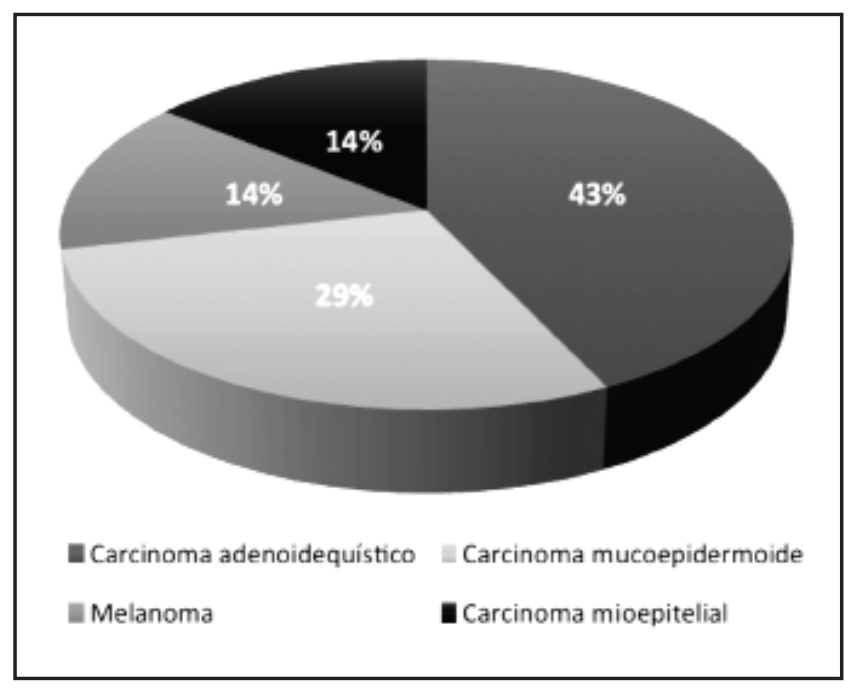

Figura 2. Histología tumores malignos de glándula submandibular. 
Tabla 1. Cuadro comparativo entre patología tumoral benigna y maligna de glándula submandibular

\begin{tabular}{|lll|}
\hline & Benignos & Malignos \\
\hline Edad & 53 años & 57 años \\
Relación M:H & $2: 1$ & $6: 1$ \\
Tiempo cirugía & 93 min & 114 min \\
Días con drenaje & 2 días & 2,14 días \\
Duración estadía posoperatoria & 2,2 días & 2,14 días \\
Complicaciones & 1 caso & 1 caso \\
Seguimiento & 15 meses & 39 meses \\
\hline Total & 21 & 7 \\
\hline
\end{tabular}

Celedón y cols. (2002), publicaron su experiencia de 20 años en tumores de glándulas salivales, siendo los tumores de glándula submandibular el $6 \%$ (8 pacientes) ${ }^{18}$. En 2005, el Instituo Nacional del Cáncer presentó su experiencia de 12 años en tumores de glándulas salivales, correspondiendo el $16 \%$ (16 pacientes) a tumores submandibulares ${ }^{19}$. En nuestro centro los tumores de glándula submandibular corresponden al $22 \%$ de los tumores de glándulas salivales, lo que representa un porcentaje más alto al reportado clásicamente.

A diferencia de los tumores parotídeos, en que el $80 \%$ son benignos, los tumores submandibulares son malignos en aproximadamente el $50 \%{ }^{4}$. Spiro y cols, reportaron un porcentaje de malignidad del $43 \%{ }^{7}$, mientras que estudios más recientes, como el de Bradley y cols, reportan tasas más bajas del $25 \%{ }^{12}$. En nuestra serie presentamos $25 \%$ de tumores malignos, más bajo que lo reportado habitualmente, pero sí concordante con las publicaciones recientes.

Clínicamente se presentan como una masa submandibular indolora ${ }^{13}$. Esta fue la presentación en el $100 \%$ de los casos. Generalmente la presencia de dolor orienta a un proceso benigno como sialoadenitis o sialolitiasis ${ }^{13}$. En nuestro centro, los 15 pacientes que fueron excluidos de este estudio por presentar patología inflamatoria y/o litiásica, consultaron por dolor e induración de la región submandibular, mientras que los 28 incluidos en este estudio por presentar patología tumoral no presentaron dolor. En cuanto a los signos de malignidad, se describe la presencia de parálisis de nervio lingual, hipogloso y/o marginal, hallazgo que no estuvo presente en nuestra serie ${ }^{13}$.
La evaluación preoperatoria de estos pacientes permite orientar el diagnóstico y el manejo, pero el diagnóstico definitivo se realiza con la biopsia de la pieza operatoria ${ }^{14-17}$. Los estudios solicitados en nuestro centro son la ecografía y la TC con contraste, a pesar de disponer de resonancia magnética (RM), el cual es el examen de elección para diferenciar entre tumores benignos y malignos y para evaluar la extensión del tumor, este examen no fue solicitado debido a que retrasaba la cirugía por falta de disponibilidad ${ }^{14}$. Sin embargo, sí fue solicitada la RM en los casos con recidiva local, en los casos de recurrencia la RM también es el examen de elección ${ }^{14}$.

La punción aspirativa con aguja fina es controversial, se ha reportado una sensibilidad de $97 \%$ con especificidad de $80 \%$ y $20 \%$ de falsos negativos ${ }^{15,21}$. En nuestro centro no es utilizada en tumores de glándulas salivales al no contar con un patólogo especializado, sólo se ha usado en forma esporádica en tumores parotídeos. Otra alternativa es la biopsia core guiada por ecografía, la cual tiene una sensibilidad de $96 \%$, especificidad de $100 \%$ y en $1,6 \%$ es no diagnóstica (versus $8 \%$ de la PAAF) según un metaánálisis reciente ${ }^{22}$. En nuestro centro, esta técnica está disponible y se ha realizado en algunos casos seleccionados de tumores parotídeos, pero no en tumores de glándula submandibular.

Como se mencionó previamente, los tumores submandibulares son malignos en el $50 \%$ de los $\operatorname{cas}^{4,7}$. La experiencia nacional presenta resultados similares, tanto en la serie de Celedón y cols., y la de Madrid y cols, el $50 \%$ de los tumores submandibulares fueron malignos (4 y 13 casos, 
respectivamente) $)^{18,20}$. Sin embargo, publicaciones más recientes, como la de Tian y cols, y la de Bradley y cols, reportan que el $26 \%$ y $25 \%$ de los casos respectivamente, corresponden a tumores malignos, resultados similares a los de nuestra casuística ${ }^{10-12}$.

El tumor benigno más frecuente es el adenoma pleomorfo, dato que se repite en los dos trabajos chilenos publicados a la fecha y en nuestra se$r^{18-20}$. En nuestra experiencia, el segundo tumor benigno más frecuente fue el tumor de Küttner 0 sialoadenitis crónica esclerosante, lesión pseudotumoral poco frecuente que afecta predominantemente a las glándulas submandibulares que se caracteriza por la aparición de una masa indolora de crecimiento lento y progresivo ${ }^{23}$. También presentamos un tumor solitario fibroso, patología infrecuente de carácter benigno de origen mesenquimal localizado principalmente en parótida, y otro caso de adenoma de células basales, que corresponde a un adenoma monomorfo, muy infrecuente localizado principalmente en parótida ${ }^{24,25}$. En cuanto a los tumores malignos, las variantes más frecuentes son el carcinoma adenoidequístico y el carcinoma mucoepidermoide, lo cual fue concordante en nuestra serie ${ }^{4,17}$.

La submandibulectomía es una técnica segura con baja tasa de complicaciones, siendo las más frecuentes la hemorragia (0\%-14\%), infección de la herida operatoria $(0 \%-14 \%)$, parálisis facial transitoria $(10 \%)$, fístula $(0 \%-4 \%)$, parálisis permanente del nervio facial $(3 \%)$, lingual $(2 \%)$ e hipogloso $(1 \%)^{16}$. En nuestra serie presentamos dos complicaciones transitorias $(7 \%)$, una parálisis transitoria del nervio marginal $(3,5 \%)$ y otra del nervio hipo-

\section{BIBLIOGRAFÍA}

1. Ferrer C, Ramos V, Ferrer E, Sancho R. The rol of radioteraphy in the manegement of salivary gland neoplasms. Medicina Oral 1998; 3: 20721.

2. WITT RL. Major salivary gland cancer. Surg Oncol Clin N Am 2004; 13: 113-27.

3. Horn-Ross PL, West DW, Brown SR. Recent trends in the incidence of salivary gland cancer. Int J Epidemiol 1991; 20: 628-33.

4. Bradley PJ. Frequency and Histopathology by gloso (3,5\%), ambas dentro del porcentaje esperado de complicaciones. Respecto a la recurrencia, presentamos tres casos de recurrencia (43\%), siendo lo reportado alrededor del $45 \%{ }^{13}$. Nuestra sobrevida a 36 meses es de $100 \%$ en patología maligna, pero cabe destacar que actualmente dos de esos tres pacientes se encuentran en cuidados paliativos con metástasis a distancia.

Nuestra serie presenta resultados acordes a la literatura en cuanto a edad de presentación, distribución por sexo, tipos histológicos, tasa de complicaciones y recurrencia; sin embargo, difiere en la mayor frecuencia de tumores malignos en mujeres (relación 6:1), hallazgo también reportado por Madrid y cols ${ }^{19}$.

\section{CONCLUSIÓN}

Los tumores de glándula submandibular son una patología poco frecuente caracterizada por la presencia de una masa indolora submandibular entre la $5^{\mathrm{a}}-6^{\mathrm{a}}$ década de la vida. El estudio preoperatorio no logra diferenciar claramente entre patología tumoral benigna y maligna, por lo que es fundamental realizar estudio histológico, siendo la submandibulectomía el tratamiento de elección, técnica segura con baja tasa de complicaciones que permite el diagnóstico definitivo. La presencia de una masa indolora submandibular debe ser derivada a los centros de referencia para realizar un manejo adecuado por el riesgo de malignidad que conlleva. Nuestra serie, si bien es pequeña, presenta resultados concordantes con la literatura y es una de las más grandes de nuestro país.

Site, Major Pathologies, Symptoms and Signs of Salivary Gland Neoplasms. Adv Otorhinolaryngol 2016; 78: 9-16.

5. ThaCkRAY AC. Salivary gland tumours - pathology. Proc Roy Soc Med 1968; 61: 1089-92.

6. Eveson JW, Cawson RA. Salivary gland tumours. A review of 2,410 cases with reference to histological types, site, age and sex distribution. J Pathol 1985; 146: 51-8.

7. SpIRo RH. Salivary neoplasms: Overview of a 35 year experience with 2,807 patients. Head Neck Surg 1986; 8: 177-84. 
8. Jones AV, Craig GT, Speight PM, et al. The range and demographics of salivary gland tumours diagnosed in a UK population. Oral Oncol 2008; 44: 407-17.

9. LI LJ, LI Y, WEN YM, ET AL. Clinical anal- ysis of salivary gland tumour cases in West China in past 50 years. Oral Oncol 2008; 44: 187-92.

10. TIAN Z, LI L, Wang L, ET AL. Salivary gland neoplasms in oral and maxillofacial regions: a 23-year retrospective study of 6982 cases in an Eastern Chinese population. Oral Maxillofac Surg 2010; 39: 235-42.

11. SpIRo RH. Management of malignant tumours of the salivary glands. Oncology 1998; 12: 671-83.

12. Bradley PJ, McGurk M. Incidence of salivary gland neoplasms in a define UK population. $\mathrm{Br}$ J Oral Maxillofac Surg 2013; 51: 399-403.

13. Weber RS, Byers RM, Petit B, et al. Submandibular gland tumours, adverse histologic factors and therapeutic implications. Arch Otolaryngol Head Neck Surg 1990; 116: 105560.

14. Kontzialis M, Glastonbury C, Aygun N. Evaluation: Imaging studies. Adv Otorhinolaryngo/2016; 78 : 25-38.

15. Howlett DC, Triantafyllou A. Evaluation: Fine Needle Aspiration Cytology, Ultrasound-Guided Core Biopsy and Open Biopsy Techniques. Adv Otorhinolaryngol 2016; 78: 39-45.

16. GILLESPIE MB, IRO H. Surgery for benign salivary neoplasms. Adv Otorhinolaryngol 2016; 78: 5362.

17. Silver NL, Chinn SB, Bradley PJ, Weber RS. Surgery for malignant submandibular gland neoplasms. Adv Otorhinolaryngol 2016; 78 : 104-12.
18. Celedón C, Ojeda J, Agurto M, Olavarría C, Niklischek E, Paredes A. Tumores de glándulas salivales. Experiencia de 20 años. Rev Otorrinolaringol Cir Cabeza Cuello 2002; 62: 255-64.

19. Larraín D, Madrid A, Capdevillle F, Ferrada C. Tumores de glándulas salivales: Hallazgos histopatológicos en 168 pacientes. Rev Chil Cir 2005; 57 : 373-8.

20. Madrid A, Larraín D, Capdeville F, Ferrada C, Cumsille M. Tumores de la glándula submandibular: Experiencia del Instituto Nacional del Cáncer. Rev Chil Cir 2005; 57: 19-25.

21. Schmidt RL, Hall BJ, Wilson AR, et al. A systematic review and meta-analysis of the diagnostic accuracy of fine needle aspiration cytology for parotid gland lesions. Am J Clin Pathol 2011; 136: 45-59.

22. Witt BL, Schmidt RL. Ultrasound-guided core needle biopsy of salivary gland lesions: a systematic review and meta-analysis. Laryngoscope 2014; 124: 695-700.

23. Martínez N, Baquera J, Sánchez R, Márquez M, Ortíz Hidalgo C. Tumor de Küttner (sialoadenitis crónica esclerosante). Estudio clinicopatológico e inmunohistoquímico de 8 casos de una entidad poco reconocida. Acta Otorrinolaringol Esp 2007; 58: 25-30.

24. Martínez V, Zarraonandia I, Monzón M, Ferrer D. Tumor fibroso solitario de la glándula submandibular. Acta Otorrinolaringol Esp 2015; 66: 302-4.

25. González R, Nam S, Muñoz M, Gamallo A. Adenoma de células basales de glándula parótida: Caso clínico y revisión de la literatura. Med Oral Pato Oral Cir Bucal 2006; 11: 206-9. 